\section{Prevalence and management of iron overload in pyruvate kinase deficiency: report from the Pyruvate Kinase Deficiency Natural History Study}

Pyruvate kinase (PK) deficiency is the most common red cell glycolytic enzyme defect causing hereditary nonspherocytic hemolytic anemia. Current treatments are mainly supportive and include red cell transfusions and splenectomy. ${ }^{1}$ Regular red cell transfusions are known to result in iron overload; however, the prevalence and spectrum of transfusion-independent iron overload in the overall PK-deficient population has not been well defined. This analysis describes the prevalence and clinical characteristics of iron overload in patients enrolled in the PK Deficiency Natural History Study (NHS) with a focus on those patients who are not regularly transfused. ${ }^{2}$

The PK deficiency NHS protocol (clinicaltrials.gov identifier: 02053480) was approved by each site's Institutional Review Board (IRB) and/or Ethics Committee, and study procedures were in accordance with the Declaration of
Helsinki. In Lancaster, Pennsylvania, USA, in which all enrolled patients are Amish, additional laboratory and radiological data were collected under a site-specific IRBapproved protocol. All patients gave informed consent. The NHS enrolled 278 patients with PK deficiency from June 2014 through April 2017 at 31 centers in 6 countries. A detailed description of the cohort is published elsewhere. ${ }^{2}$ Twenty-four patients were excluded due to the inability to confirm two pathogenic PKLR mutations. Patients under one year old at enrollment were also excluded $(n=12)$ from this analysis, because ferritin is less reliably related to iron overload in this youngest age group, leaving 242 participants reported herein. Patients were defined as regularly transfused if they had received $\geq 6$ transfusions in the 12 months prior to enrollment. At enrollment, $82 \%(198 / 242)$ of patients were not receiving regular transfusions; $38 \%(53 / 138)$ of these patients had iron overload as defined by ferritin. The patients with iron overload were older, more anemic, and more often splenectomized, and had a higher median total bilirubin (Table 1).

Table 1. Characteristics of non-regularly transfused patients with pyruvate kinase (PK) and iron overload.

\begin{tabular}{|c|c|c|c|c|c|c|}
\hline & Ferritin & 1000 ng/mL or & ion* & LIC $>3$ & dry weight live & ation* \\
\hline & $\begin{array}{c}\text { Absent } \\
(85 / 138,62 \%)\end{array}$ & $\begin{array}{c}\text { Present } \\
(53 / 138,38 \%)\end{array}$ & $P^{* *}$ & $\begin{array}{c}\text { Absent } \\
(15 / 82,18 \%)\end{array}$ & $\begin{array}{c}\text { Present } \\
(67 / 82,82 \%)\end{array}$ & $P^{* *}$ \\
\hline Female sex & 49/85 (58\%) & $24 / 53(45 \%)$ & 0.2 & $12 / 15(80 \%)$ & $36 / 67(54 \%)$ & 0.08 \\
\hline Amish & $27 / 85(32 \%)$ & $13 / 53(25 \%)$ & 0.4 & $14 / 15(93 \%)$ & $33 / 67(49 \%)$ & 0.001 \\
\hline Age at enrollment (y) & $\begin{array}{c}22.6 \\
(1.6-69.9)\end{array}$ & $\begin{array}{c}38.9 \\
(2.2-60.4)\end{array}$ & 0.005 & $\begin{array}{c}23.4 \\
(7.4-53.6)\end{array}$ & $\begin{array}{c}34.7 \\
(3.1-60.4)\end{array}$ & 0.3 \\
\hline Hemoglobin (g/dL) & $\begin{array}{c}9.3 \\
(6.2-14.1)\end{array}$ & $\begin{array}{c}8.7 \\
(6.5-12.0) \\
n=52\end{array}$ & 0.003 & $\begin{array}{c}9.9 \\
(7.6-10.9)\end{array}$ & $\begin{array}{c}9.0 \\
(6.2-12.0) \\
n=66\end{array}$ & 0.2 \\
\hline
\end{tabular}

\begin{tabular}{|c|c|c|c|c|c|c|}
\hline Absolute reticulocyte count $\left(10^{6} / \mathrm{L}\right)$ & $\begin{array}{c}0.2 \\
(0.1-5.3) \\
n=41\end{array}$ & $\begin{array}{c}0.5 \\
(0.1-1.2) \\
n=15\end{array}$ & 0.2 & $\begin{array}{c}0.9 \\
(0.9-1.0) \\
n=2\end{array}$ & $\begin{array}{c}0.6 \\
(0.1-0.9) \\
n=13\end{array}$ & 0.1 \\
\hline Total bilirubin (mg/dL) & $\begin{array}{c}3.6 \\
(0.9-9.0) \\
n=79\end{array}$ & $\begin{array}{c}4.3 \\
(1.3-17.6) \\
n=48\end{array}$ & 0.01 & $\begin{array}{c}3.5 \\
(1.0-7.2)\end{array}$ & $\begin{array}{c}3.6 \\
(1.1-17.6) \\
n=62\end{array}$ & 0.3 \\
\hline Ferritin (ng/mL) & $\begin{array}{c}388.0 \\
(31.0-971.5)\end{array}$ & $\begin{array}{c}1335.0 \\
(171.5-5630.0) \\
n=47\end{array}$ & ND & $\begin{array}{c}362.5 \\
(126.0-1065.0) \\
n=12\end{array}$ & $\begin{array}{c}969.0 \\
(171.5-5630.0) \\
n=53\end{array}$ & $<0.001$ \\
\hline $\begin{array}{l}\text { Liver iron concentration } \\
\text { (mg/g DW) }\end{array}$ & $\begin{array}{c}4.0 \\
(1.0-8.4) \\
n=26\end{array}$ & $\begin{array}{c}8.0 \\
(2.2-33.4) \\
n=21\end{array}$ & $<0.001$ & $\begin{array}{c}2.0 \\
(1.0-3.0)\end{array}$ & $\begin{array}{c}6.4 \\
(2.2-33.4) \\
n=43\end{array}$ & ND \\
\hline Transferrin saturation (\%) & $\begin{array}{c}43.3 \\
(8.8-100.0) \\
n=51\end{array}$ & $\begin{array}{c}62.0 \\
(18.4-100.0) \\
n=29\end{array}$ & 0.02 & $\begin{array}{c}42.4 \\
(23.2-96.6)\end{array}$ & $\begin{array}{c}50.1 \\
(12.1-100.0) \\
n=43\end{array}$ & 0.3 \\
\hline Splenectomized & $51 / 85(60 \%)$ & $47 / 53(89 \%)$ & 0.0002 & $14 / 15(93 \%)$ & $63 / 67(94 \%)$ & 1 \\
\hline Chelation in prior 12 months & $0 / 85(0 \%)$ & $31 / 53(58 \%)$ & ND & 0/15 (0\%) & $31 / 67(46 \%)$ & ND \\
\hline Never transfused & $18 / 85(21 \%)$ & $4 / 51(8 \%)$ & 0.054 & $1 / 15(7 \%)$ & $6 / 65(9 \%)$ & 1 \\
\hline$<10$ lifetime transfusions & $22 / 61(36 \%)$ & $7 / 39(18 \%)$ & 0.07 & $2 / 9(22 \%)$ & $9 / 51(18 \%)$ & 0.7 \\
\hline$\geq 10$ lifetime transfusions & $39 / 61(64 \%)$ & $32 / 39(82 \%)$ & 0.07 & $7 / 9(78 \%)$ & $42 / 51(82 \%)$ & 0.7 \\
\hline
\end{tabular}

Numbers are medians (range) or absolute numbers/group total (corresponding percentage). $n$ is shown only for those groups that do not contain the total. *Iron overload by ferritin was defined as the number of patients with ferritin>1000 ng/mL or who were treated with chelation therapy in the 12 months prior to enrollment. If a patient had $>1$ ferritin measurement in the 12 months prior to enrollment, the maximum ferritin value was used. Iron overload based on liver iron concentration (LIC) was defined as an LIC $>3 \mathrm{mg}$ Fe/g dry weight liver (DW) on T2* MRI in the 12 months prior to enrollment or who were treated with chelation therapy in the 12 months prior to enrollment. ${ }^{* *} P$-values of Fisher's Exact test or Wilcoxon Rank Sum test. ND: Not Done, testing is not appropriate or necessary within the same factor. 
Baseline and retrospective clinically available data were used. Serum ferritin levels and quantitative (T2*) liver and heart magnetic resonance imaging (MRI) were used to assess iron overload. Patients were considered to have iron overload if: i) their highest ferritin was over 1000 $\mathrm{ng} / \mathrm{mL}$; or ii) they received chelation therapy in the 12 months prior to enrollment; or iii) their highest liver iron concentration (LIC) was $>3 \mathrm{mg} / \mathrm{g}$ dry weight liver (DW) on T2* MRI; or iv) they had cardiac iron overload as defined by a cardiac T2* $\leq 20 \mathrm{~ms}$ at any time in their history. The definition of iron overload as an LIC $>3 \mathrm{mg} / \mathrm{g}$ DW was based on guidelines for $\beta$-thalassemia. ${ }^{3,4}$

Baseline characteristics and results are listed in Table 1. Of the 242 patients, 175 (72\%) had ferritin levels measured within the prior 12 months. The median ferritin was $583 \mathrm{ng} / \mathrm{mL}$ (range, 17-5630 $\mathrm{ng} / \mathrm{mL}$ ). The overall prevalence of iron overload as defined by ferritin or chelation was $45 \%$ (82/181). Patients without ferritin monitoring had fewer transfusions ( $1 \%$ vs. $25 \%$ regularly transfused; $P<0.0001)$ and a higher hemoglobin $(\mathrm{Hb})$ level (median $\mathrm{Hb} 9.6$ vs. $8.8 \mathrm{~g} / \mathrm{dL} ; P=0.01$ ) (Online Supplementary Table S1).

An MRI for liver iron assessment was conducted in 65 $(27 \%)$ patients in the 12 months prior to enrollment; 47 $(72 \%)$ were from the Amish cohort and were obtained per protocol. Of the patients who were not receiving regular transfusions who had MRI or chelation data available, $82 \%(67 / 82)$ had iron overload as defined by MRI or chelation (Table 1). Of those patients who had never been transfused and had MRI or chelation data available, 6 of 7 patients met criteria for iron overload ( $6 / 7$ splenectomized).

Magnetic resonance imaging for cardiac iron assessment was available for $75(31 \%)$ patients. Five patients $(7 \%)$ had cardiac iron overload; only one of these patients had LIC measured (5 mg Fe/g DW). These patients' ages ranged from 3-34 years with a median number of lifetime transfusions of 39 (range, 10-90).

There were 68 patients aged $1-<10$ years old. Of those who were not regularly transfused at enrollment but had received occasional transfusions, $82 \%(9 / 11)$ had iron overload as defined by MRI. The median number of lifetime transfusions in this group was 22.5 (range, 11-85). Of those under 10 years of age who had never been transfused $(n=9)$, MRI was available for only one patient, which confirmed iron overload.

Forty-five patients had paired ferritin and LIC measurements available (Figure 1). Using a ferritin cut off of 1000 $\mathrm{ng} / \mathrm{mL}$, the sensitivity to predict LIC $>3 \mathrm{mg} / \mathrm{g}$ DW was $53 \%$ and the specificity was $100 \%$. At a ferritin cut off of $500 \mathrm{ng} / \mathrm{mL}$, the sensitivity for LIC $>3 \mathrm{mg} / \mathrm{g}$ DW was $90 \%$ and the specificity was $67 \%$.

Of the 242 patients, 82 (34\%) had been prescribed chelation therapy during their lifetime. The median age at the time chelation therapy was first initiated was 10.4 years (range, 0.7-47.9 years). Of those who had never been transfused, $10 \%$ had received chelation therapy. Of those patients aged $1-<10$ years at enrollment, $19 \%$ had been on chelation therapy starting at a median age of 2.4 years (range, $2-5$ years).

Disease treatment of Amish patients differed significantly from the other patients, as these patients are typically uninsured and the cost of standard supportive care can be prohibitive. All but 2 Amish patients were splenectomized; none of the splenectomized patients received regular transfusions. Instead of chelation therapy, Amish patients were managed with an iron restricted diet, and a combination of proton pump inhibitors and calcium citrate to reduce dietary iron uptake.

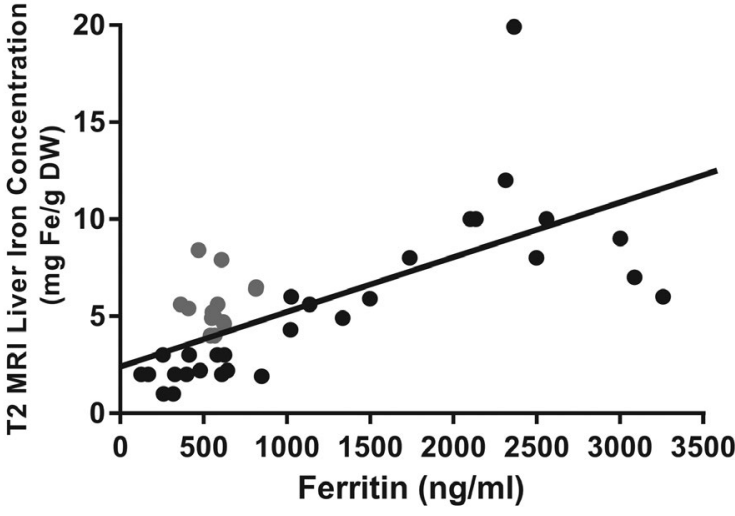

Figure 1. Correlation between ferritin and liver iron concentration (LIC) as measured by magnetic resonance imaging. Correlation between ferritin and LIC $(r=0.45, P<0.0001 ; n=45)$. Gray circles indicate individuals with a median ferritin $<1000 \mathrm{ng} / \mathrm{mL}$ but an LIC $>3 \mathrm{mg} / \mathrm{g}$ dry weight liver.

In this PK deficiency Natural History Study, the prevalence of iron overload defined by LIC was $82 \%$ in nonregularly transfused patients. Although ferritin levels correlated with LIC, ferritin levels of $>1000 \mathrm{ng} / \mathrm{mL}$ had a sensitivity for LIC $>3 \mathrm{mg} / \mathrm{g}$ DW of only $53 \%$. A ferritin $>1000 \mathrm{ng} / \mathrm{mL}$ is a conservative threshold for reporting the prevalence of iron overload given the specificity of $100 \%$ but likely underestimates its true prevalence in PK deficiency. Given that the ferritin $>500 \mathrm{ng} / \mathrm{mL}$ had sensitivity of $90 \%$, this is a better cut off if ferritin is used as a screening test for selecting patients for an MRI.

Despite the conservative ferritin threshold of $>1000$ $\mathrm{mg} / \mathrm{dL}, 38 \%$ of all patients who were not regularly transfused and $18 \%$ of those who were never transfused met this definition of iron overload. Furthermore, $82 \%$ of the patients who were not regularly transfused and had an MRI or chelation data had LIC $>3 \mathrm{~g} / \mathrm{mg}$ DW. These data clearly show that iron overload is not limited to regularly transfused patients but is also common in patients who are not regularly transfused and even in those who have never been transfused. This is consistent with the findings in thalassemia intermedia in which iron loading occurs both in transfused and non-transfused patients.

There is no consensus on a definition of when patients with PK deficiency are regarded as regularly transfused. Factors influencing transfusion frequency are often patient or physician dependent. Transfusion triggers vary from hospital to hospital and the degree of transfusion dependence of the patient might also differ based on characteristics such as age, growth, and daily activities. By choosing a conservatively high cut off of at least 6 transfusions per year to distinguish between regularly and not-regularly transfused patients, we minimized the chance of confounding transfusion-related iron loading with transfusion-independent iron overload.-7

In this study, in non-regularly transfused patients, iron overload had also already occurred at a very young age. In non-regularly transfused patients, an MRI should be considered at the earliest age when the procedure can be performed without sedation, particularly in patients with ferritin levels $>500 \mathrm{ng} / \mathrm{mL}$. In regularly transfused patients, MRI should be considered annually after one year of transfusions.

In this cohort, only 5 patients $(7 \%)$ had cardiac iron overload by MRI. One patient in the cohort developed 
cardiac iron overload after only 10 lifetime transfusions. Moreover, the youngest patient with cardiac iron overload in this cohort was three years old. This indicates that the number of prior transfusions might not predict which patients are most in need of screening for cardiac iron overload.

In the non-Amish cohort, splenectomy was associated with iron loading. However, this association is not clearly causal. Since splenectomy typically occurs in the more severely affected patients with PK deficiency, other factors associated with splenectomy, such as a lower $\mathrm{Hb}$ or increased transfusion burden, may be contributing to this relationship. Further study is needed to understand whether splenectomy is independently associated with iron loading in PK deficiency.

Although the study is biased by its retrospective nature and challenges related to rare disease registries, including variability between diagnostic and treatment standards, the results clearly show that in PK deficiency, there is a high prevalence of iron overload both in regularly transfused and not regularly transfused patients. Ferritin levels below $1000 \mathrm{ng} / \mathrm{mL}$, Hb levels above 9 g/dL, young age, and/or an absence of regular transfusions do not exclude the possibility of iron overload in patients with PK deficiency. Therefore, iron screening is important in all patients with PK deficiency and should be monitored starting in childhood. Regular monitoring for iron overload and treatment, when needed, is imperative to the management of this patient population.

Eduard J. van Beers, ${ }^{1}$ Stephanie van Straaten, D. Holmes Morton, ${ }^{2}$ Wilma Barcellini, ${ }^{3}$ Stefan W. Eber, ${ }^{4}$ Bertil Glader, Hassan M. Yaish, ${ }^{5}$ Satheesh Chonat, ${ }^{7}$ Janet L. Kwiatkowski, Jennifer A. Rothman," Mukta Sharma, ${ }^{10}$ Ellis J. Neufeld, ${ }^{11}$ Sujit Sheth, ${ }^{12}$ Jenny M. Despotovic, ${ }^{13}$ Nina Kollmar, ${ }^{14}$ Dagmar Pospiśilová, ${ }^{15}$ Christine M. Knoll, ${ }^{16}$ Kevin Kuo, ${ }^{17}$ Yves D. Pastore, ${ }^{18}$ Alexis A. Thompson, ${ }^{19}$ Peter E. Newburger, ${ }^{20}$ Yaddanapudi Ravindranath, ${ }^{21}$ Winfred C. Wang, ${ }^{11}$ Marcin W. Wlodarski, ${ }^{22}$ Heng Wang, ${ }^{23}$ Susanne Holzhauer, ${ }^{24}$ Vicky R. Breakey, ${ }^{25}$ Madeleine Verhovsek, ${ }^{25}$ Joachim Kunz, Melissa A. McNaull, ${ }^{27}$ Melissa J. Rose, ${ }^{28}$

Heather A. Bradeen, ${ }^{29}$ Kathryn Addonizio, ${ }^{30}$ Anran Li, ${ }^{30}$ Hasan Al-Sayegh, ${ }^{30}$ Wendy B. London ${ }^{30}$ and Rachael F. Grace

'Van Creveldkliniek, University Medical Centre Utrecht, University of Utrecht, the Netherlands; ${ }^{2}$ Central Pennsylvania Clinic for Special Children \& Adults, Belleville, PA, USA; Lancaster General Hospital, Lancaster, PA, USA; ${ }^{3}$ Fondazione IRCCS Ca' Granda, Ospedale Maggiore Policlinico, Milan, Italy; ${ }^{4}$ Schwerpunktpraxis für Pädiatrische Hämatologie-Onkologie and Children's Hospital, Technical University, Munich, Germany; 'Lucile Packard Children's Hospital, Stanford University, Palo Alto, CA, USA; 'Primary Children's Hospital, University of Utah, Salt Lake City, UT, USA, ' Emory University School of Medicine, Aflac Cancer and Blood Disorders Center, Children's Healthcare of Atlanta, GA, USA; ${ }^{8}$ Children's Hospital of Philadelphia and Perelman School of Medicine of the University of Pennsylvania, Philadelphia, PA, USA; ${ }^{\circ}$ Duke University Medical Center, Durham, NC, USA; ${ }^{10}$ Children's Mercy Hospital, University of Missouri, Kansas City, MO, USA; "'St. Jude Children's Research Hospital, Memphis, TN, USA; ${ }^{12}$ Weill Cornell Medical College, New York Presbyterian Hospital, NY, USA; ${ }^{13}$ Texas Children's Hematology Center, Baylor College of Medicine, Houston, TX, USA; ${ }^{14}$ Klinikum Kassel GmbH, Germany; ${ }^{15}$ Fakultni nemocnice Olomouc, Czech Republic; ${ }^{16}$ Phoenix Children's Hospital, AZ, USA; ${ }^{17}$ University of Toronto, University Health Network, ON, Canada; ${ }^{18} \mathrm{CHU}$ SainteJustine, Montreal, QC, Canada; ${ }^{19}$ Ann \& Robert H. Lurie Children's Hospital of Chicago, Chicago, IL, USA; ${ }^{20}$ University of Massachusetts Medical School, Worcester, MA, USA; ${ }^{21}$ Children's Hospital of Michigan, Wayne State University School of Medicine, Detroit, MI, USA; ${ }^{22}$ Department of Pediatrics and Adolescent Medicine, Division of Pediatric Hematology and Oncology, Medical Center, Faculty of Medicine, University of Freiburg, Germany; ${ }^{23} D D C$ Clinic for Special Needs Children, Middlefield, OH, USA; ${ }^{24}$ Charite, Berlin, Germany; ${ }^{25}$ McMaster University, Hamilton, ON, Canada; ${ }^{26}$ Zentrum für Kinder-und Jugendmedizin, University of Heidelberg, Heidelberg, Germany; ${ }^{27}$ University of Mississippi Medical Center, Jackson, MS, USA; ${ }^{28}$ Nationwide Children's Hospital, The Ohio State University College of Medicine, Columbus, OH, USA; ${ }^{29}$ The University of Vermont Children's Hospital, Burlington, VT, USA and ${ }^{30}$ Dana-Farber Boston Children's Cancer and Blood Disorder Center, Boston, MA, USA

Correspondence: rachael.grace@childrens.harvard.edu doi:10.3324/haematol.2018.196295

Acknowledgments: the authors would like to thank all the patients with pyruvate kinase deficiency and their family members who contributed data to this natural history study. For their work on the molecular analysis, the authors thank Paola Bianchi, Elisa Fermo, Patrick Gallagher, and Kimberly Lezon-Geyda.

Information on authorship, contributions, and financial \& other disclosures was provided by the authors and is available with the online version of this article at www. haematologica.org.

\section{References}

1. Grace RF, Zanella A, Neufeld EJ, et al. Erythrocyte pyruvate kinase deficiency: 2015 status report. Am J Hematol. 2015;90(9):825-830.

2. Grace RF, Bianchi P, van Beers EJ, et al. The clinical spectrum of pyruvate kinase deficiency: data from the Pyruvate Kinase Deficiency Natural History Study. Blood. 2018;131(20):2183-2192.

3. Brittenham GM. Iron-chelating therapy for transfusional iron overload. N Engl J Med. 2011;364(2):146-156.

4. Saliba AN, Harb AR, Taher AT. Iron chelation therapy in transfusiondependent thalassemia patients: current strategies and future directions. J Blood Med. 2015;6:197-209.

5. Origa R, Galanello R, Ganz T, et al. Liver iron concentrations and urinary hepcidin in beta-thalassemia. Haematologica. 2007;92(5):583588.

6. Piga A, Galanello R, Forni GL, et al. Randomized phase II trial of deferasirox (Exjade, ICL670), a once-daily, orally-administered iron chelator, in comparison to deferoxamine in thalassemia patients with transfusional iron overload. Haematologica. 2006;91(7):873-880.

7. Cappellini MD, Porter J, El-Beshlawy A, et al. Tailoring iron chelation by iron intake and serum ferritin: the prospective EPIC study of deferasirox in 1744 patients with transfusion-dependent anemias. Haematologica. 2010;95(4):557-566. 